\title{
Right atrial mass following transcatheter radiofrequency ablation for recurrent atrial fibrillation: thrombus, endocarditis or mixoma?
}

\author{
Massa atriale destra dopo ablazione transcatetere \\ a radiofequenza per fibrillazione atriale ricorrente: \\ trombo,endocardite o mixoma?
}

\author{
Roberta Ancona1, Salvatore Comenale Pinto1, Pio Caso1, Vito Di Palma1, \\ Francesca Pisacane1, Alfonso Roberto Martiniello1, Cesare Quarto2, \\ Nicla De Rosa3 ${ }^{3}$ Carlo Pisacane ${ }^{1}$, Raffaele Calabrò 4
}

\begin{abstract}
Right atrial mass following transcatheter radiofrequency ablation for recurrent atrial fibrillation: thrombus, endocarditis or mixoma? R. Ancona, S. Comenale Pinto, P. Caso, V. Di Palma, F. Pisacane, A.R. Martiniello, C. Quarto, N. De Rosa, C. Pisacane, R. Calabrò.

We report a case of an asymptomatic patient in whom a right atrial mass was fortuitously documented by echocardiography few months after a transcatheter radiofrequency catheter ablation for recurrent AF. No masses were seen in
\end{abstract}

the cardiac chambers before the ablative procedure, raising important diagnostic and decision-making issues. The patient was referred to the surgeon and a diagnosis of right atrial myxoma was made.

Keywords: atrial mass, atrial myxoma, right atrial thrombus, infective endocarditis, atrial fibrillation.

Monaldi Arch Chest Dis 2009; 72: 40-42.

\footnotetext{
1 Complex Operative Unit of Cardiology, Monaldi Hospital, Naples, Italy.

2 Chair of Cardiac Surgery, Second University of Naples, Monaldi Hospital, Italy.

3 Pathology Unit, Monaldi Hospital, Naples, Italy.

4 Chair of Cardiology, Second University of Naples, Monaldi Hospital, Italy.
}

\section{Introduction}

Radiofrequency (RF) catheter ablation has recently been proposed as a treatment for drug-refractory paroxysmal or permanent atrial fibrillation (AF) and has emerged as an alternative strategy to achieve sinus rhythm, in selected cases (1). However, the main limitation of conventional RF catheter ablation continues to be the risk of thromboembolism, occurring up to $2 \%$ of patients (2). Therefore, discovering an atrial mass in a patient after RF catheter ablation for AF imposes important issues of differential diagnosis (3) and decision-making. We report a case of an asymptomatic patient in whom a right atrial mass was fortuitously documented by echocardiography following transcatheter RF ablation for recurrent AF.

\section{Case report}

A 55-year-old male patient with a history of transcatheter RF ablation with trans-septal technique, performed six months earlier for recurrent $\mathrm{AF}$, was admitted for the investigation of a solid echodense right atrial mass discovered by echocar- diography in another hospital. About one month after ablation the patient had fever with shivers, lasted a few days and relapsed without antibiotic therapy. Despite the apparent effectiveness of the ablative procedure, the patient continued to have asymptomatic episodes of AF, so oral anticoagulation therapy was continued. On admission in our hospital, he appeared to be in excellent health with no major complaints. On physical examination, the patient was normotensive, without fever, and had a regular pulse rate without orthostatic changes. At the cardiovascular examination no murmurs were revealed. No jugular venous distension was observed and the findings on abdominal and pulmonary examination were unremarkable. Transthoracic echocardiography revealed a right atrial mass, near the posterior leaflet of tricuspid valve. It was large about $2.5 \times 1.8$ $\mathrm{cm}$, hyperechogenic, mobile and attached to the free wall of right atrium by a stalk (Figure 1). This image was not present at echocardiographic exam, performed before RF ablation in another hospital (we were able to examine the echocardiographic pictures the patient showed us). Transoesophageal echocardiography confirmed the right atrial mass that appeared with three lobes, with a smooth surface with- 


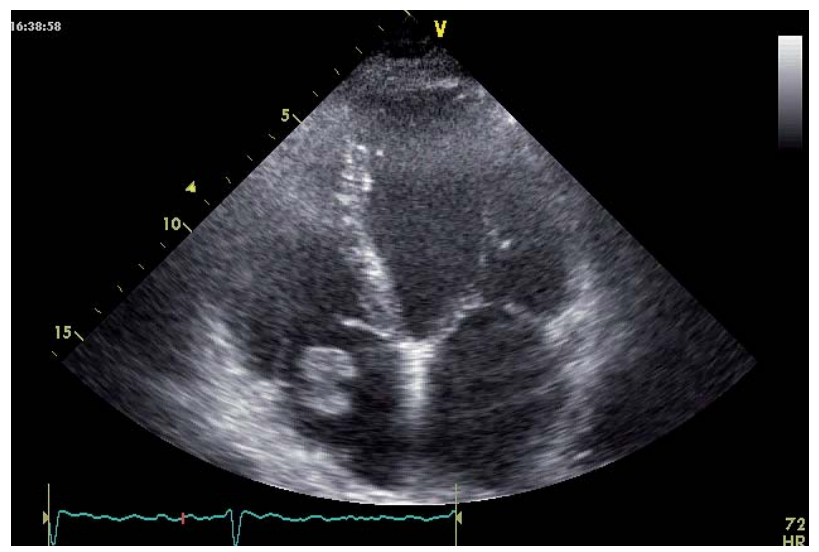

Figure 1. - Echocardiographic apical 4-chamber view. An hyperechogenic, lobate with a smooth surface right atrial mass (about $2.5 \times 1.8 \mathrm{~cm}$ ) is attached to the lateral free wall of right atrium by a stalk, near the posterior leaflet of tricuspid valve.

out involvement of tricuspid valve. The atrial mass occupied the third part of the right atrial cavity, without obstruction to the right atrial and ventricular inflow (Figure 2). Cardiac computed tomography (CT) scan confirmed a non-calcified right atrial mass attached to the free wall of right atrium without infiltration of the mural myocardium, cava veins and right ventricle. Total-body CT scan showed that all other organs appeared free from primary or secondary tumour. Multiple blood cultures were repeatedly negative. A right atriotomy was performed under total cardiopulmonary bypass and an uneventful resection of the mass was obtained. Macroscopic examination showed a $2.5 \times 2 \times 2 \mathrm{~cm}$ mass, with three lobes, with smooth but irregular surface, that appeared shiny, grey-white and brownish (Figure 3). At histology, the mass consisted mostly of a mixoid matrix with an acid-mucopolisaccaride-rich stroma including rudimentary vessels. Polygonal, starshaped and fusiform cells with scant eosinophilic cytoplasm were scattered in the ground substance. The cells presented themselves aggregated in clusters and in small cords. At immunohistochemistry

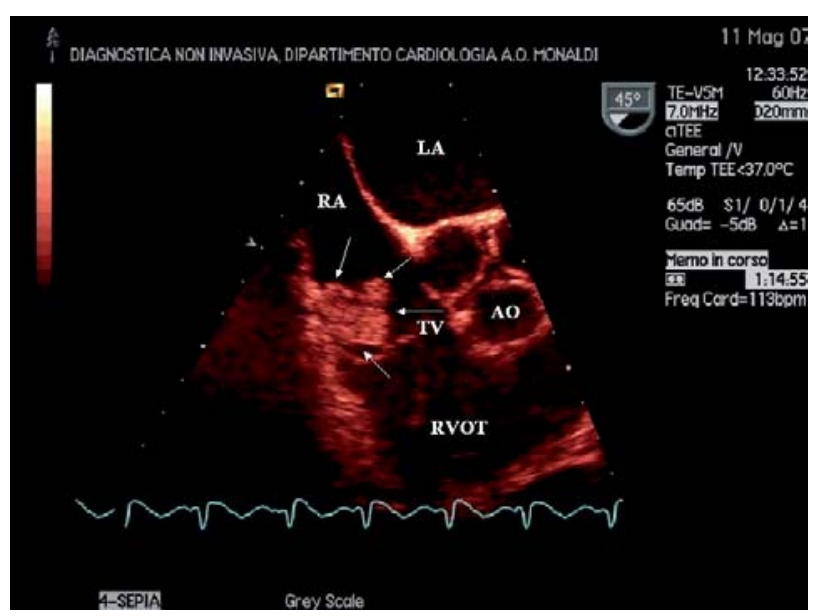

Figure 2. - Transoesophageal oblique view $\left(45^{\circ}\right)$ showing the tricuspid valve and the right ventricular outflow tract in its long axis. A three-lobated right atrial mass with a smooth surface is seen (white arrows). The atrial mass is attached to lateral wall of the right atrium and occupies the third part of the cavity without obstruction to the atrial and ventricular inflow. It is clearly separate from the tricuspid valve. $\mathrm{RA}=$ right atrium; $\mathrm{LA}=$ left atrium; $\mathrm{TV}=$ tricuspid valve; $\mathrm{AO}=$ Aortic valve; $\mathrm{RVOT}=$ right ventricle outflow tract.

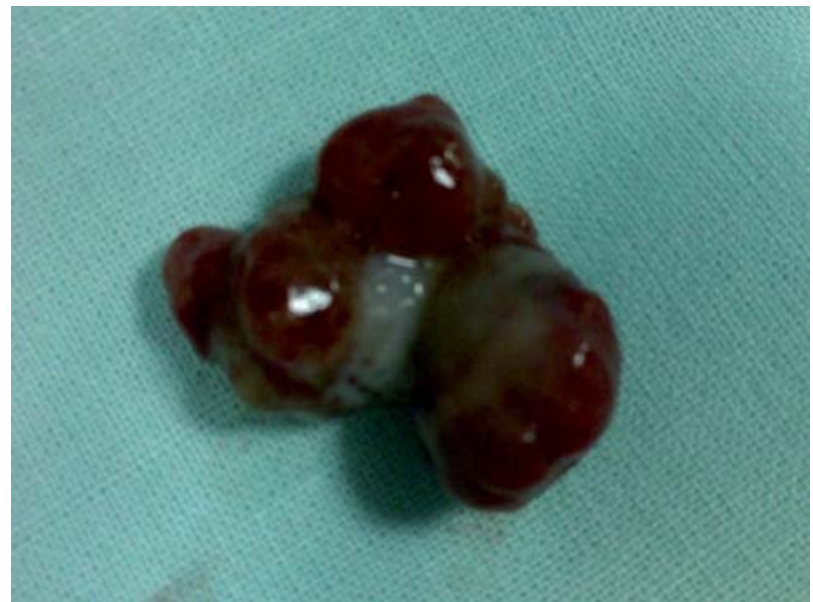

Figure 3. - Macroscopic aspect of the right atrial mass (myxoma). A three-lobated configuration is clearly evident.

the cells were cytocheratin and calretinin negative, variably S100 positive and positive for smooth muscle and endothelial markers (CD 34 and CD 31) (Figure 4). A final diagnosis of right atrial myxoma was made.

\section{Discussion}

When we found a right atrial mass by echocardiography in an asymptomatic patient after RF ablation for AF there are some possible diagnoses.

The right atrial mass was not present before admission for RV ablation. We can suspect a thrombotic complication related to the procedure. In fact, most of the literature reports that RF catheter ablation procedures are associated with an inherent risk of thrombus formation even at sites remote from ablation lesions (4), with an equal incidence for right- and left-ablations. Moreover, it is believed that thromboembolic complications occur as a result of endocardial damage secondary to catheter manipulation rather than ablation (5). Furthermore, it has been shown that thrombus formation may also be due to an activation of the coagulation cascade related to the placement of intravascular catheters and the duration of the abla-

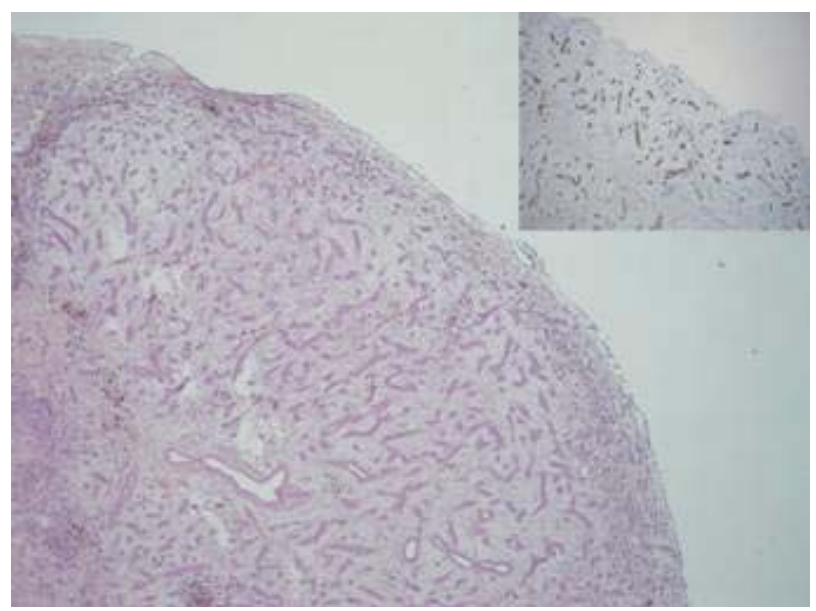

Figure 4. - Microscopic appearance of the right atrial mass. Numerous rudimentary vessels surrounded by abundant loose stroma beneath the endocardial lining are evident. Insert: The endothelial cells show a positive immunostaining for CD 34-related antigen. 
tion procedure (6). The attachment of a pedunculated mass to the lateral wall of the right atrium in this patient may support a thrombus diagnosis. However, a diagnosis of right atrial thrombosis was unlikely, because the patient was still being treated with oral anticoagulant.

On the other hand, catheters insertion in the right heart can lead to bacteraemia and endocarditis. Although infective endocarditis occurs primarily on cardiac valves, sometimes it can also involve other endocardial surfaces (mural endocardium) and at echocardiography appears as a mobile, echodense mass, frequently showing high-frequency flutter or oscillations. In this patient, however, clinical data didn't support a right atrial mural vegetation diagnosis, because no specific symptoms or laboratory findings were seen.

Moreover, tumor hypothesis has been considered.

Ninety per cent of primary cardiac tumors are either myxomas or sarcomas (7), frequently located in the right atrium. Approximately $10 \%$ of cardiac myxomas occur in the right atrium and in this location are more likely to be calcified (7). At echocardiography cardiac myxoma in the right atrium typically appears as a mobile mass attached to the endocardial surface by a stalk, that is most commonly much smaller than the maximal diameter of the mass, usually arising from the fossa ovalis (8). Sometimes, however, right atrial myxoma is attached to the atrial free wall (9).

However, exact diagnosis needs surgical excision, because myxomas and sarcomas may have similar features at echocardiography. In this patient, the right atrial mass was on lateral atrial wall, near the tricuspid valve, an unusual location for myxoma.

The growth rate of cardiac myxoma is 5.3 $\mathrm{cm} /$ year, on average (10). The right atrial mass dimensions we found were in agreement with growth in the last months. CT scan didn't show right atrial wall infiltration, as cardiac sarcomas sometimes make.

The patient was referred to the surgeon and a diagnosis of right atrial myxoma was made.

RF catheter ablation for AF increases the risk of thrombus or endocarditis in atrium. However, we have to consider unlikely diagnosis too, as mixoma, to avoid erroneous diagnosis and serious mismanagement of the patients.
This case underlines the need to consider all possible diagnostic hypothesis in patients undergoing $\mathrm{RF}$ catheter ablation for $\mathrm{AF}$, in which a right atrial mass is discovered after the procedure.

\section{Riassunto}

Viene descritto il caso di un paziente asintomatico nel quale fu riscontrata casualmente con un ecocardiogramma la presenza di una massa atriale destra pochi mesi dopo una procedura di ablazione transcatetere a $R F$ per episodi ricorrenti di FA. Il paziente fu successivamente operato e fu confermata la diagnosi di mixoma atriale destro. Poiché nessuna massa intracardiaca era presente prima della ablazione, tale riscontro solleva importanti problematiche diagnostiche e decisionali

\section{References}

1. Oral H, Pappone C, Chugh A, et al. Circumferential pulmonary-vein ablation for chronic atrial fibrillation. $\mathrm{N} \mathrm{Engl}$ J Med 2006; 354: 934-941.

2. Zhou L, Keane D, Reed G, Ruskin J. Thromboembolic complications of cardiac radiofrequency catheter ablation: a review of the reported incidence, pathogenesis and current research directions. J Cardiovasc Electrophysiol 1999; 10: 611-620.

3. Dhawan $\mathrm{S}, \mathrm{Tak} \mathrm{T}$. Left atrial mass: Thrombus mimicking myxoma. Echocardiography 2004; 21: 621-3.

4. Raitt MH, Schwaegler B, Pearlman AS, et al. Development of an aortic valve mass after radiofrequency catheter ablation. Pacing Clin Electrophysiol 1993; 16: 2064-2066.

5. Khairy P, Chauvet P, Lehmann J, et al. Lower Incidence of Thrombus Formation With Cryoenergy versus Radiofrequency Catheter Ablation. Circulation. 2003; 107: 2045-2050.

6. Dorbala S, Cohen AJ, Hutchinson LA, Menchavez-Tan E, Steinberg JS. Does radiofrequency ablation induce a prethrombotic state? Analysis of coagulation system activation and comparison to electrophysiologic study. J Cardiovasc Electrophysiol 1998; 9: 1152-60.

7. Burke A, Jeudy J JR and Virmani R. Cardiac tumors: an update. Heart 2008; 94: 117-123

8. Araoz PA, Eklund HE, Welch TJ, Breen JF. CT and MR imaging of primary cardiac malignancies. Radiographics 1999; 19: 1421-34.

9. Reynen K. Cardiac myxomas. N Engl J Med 1995; 333(24): 1610-7.

10. Pochis WT, Wingo MW, Cinquegrani MP, Sagar KB. Echocardiographic demonstration of rapid growth of a left atrial myxoma. Am Heart J 1991; 122: 1781-4. 